\title{
An active viscoelastic metamaterial for isolation applications
}

\author{
M Reynolds and S Daley \\ Institute of Sound and Vibration Research (ISVR), University of Southampton, \\ Southampton, UK, SO17 2ND \\ E-mail: matthew.reynolds@soton.ac.uk
}

\begin{abstract}
Metamaterials are of interest due to their ability to produce novel acoustic behaviour beyond that seen in naturally occurring media. Of particular interest is the appearance of band gaps which lead to very high levels of attenuation within narrow frequency ranges. Resonant elements within metamaterials allow band gaps to form within the long wavelength limit at low frequencies where traditional passive isolation solutions suffer poor performance. Hence metamaterials may provide a path to high performance, low frequency isolation. Two metamaterials are presented here. An acoustic material consisting of an array of split hollow spheres is developed and its performance validated experimentally. The application of an acoustic/mechanical analogy allows the development of an elastodynamic metamaterial that could be employed as a high performance vibration isolator at low frequencies. A prototype isolator is manufactured and its performance measured. The passively occurring band gap is enhanced using an active control architecture. The use of the active control system in conjunction with the natural passive behaviour of the metamaterial enables high levels of isolation across a broad frequency range. An eventual goal of the work is to produce such materials on a small scale, and as such the metamaterials developed are designed for, and produced using, additive layer manufacturing techniques
\end{abstract}

PACS numbers: 43.40.Vn, 43.40.Tm, 07.10.Fq, 43.20.Jr

\section{Introduction}

Over the last decade there has been significant research activity into the development of acoustic metamaterials due to the novel behaviour they produce. Locally resonant periodic materials, called metamaterials, were first proposed in the electromagnetic domain by Smith [1] as a way of realising the 'left handed' behaviour postulated by Vesalago many years earlier [2], where the behaviours of a material with simultaneously negative electromagnetic permeability and permittivity were presented. The analogy between acoustic and electromagnetic waves has recently stimulated a great deal of research into acoustic metamaterials [3, 4], where the analogues of permeability and permittivity are density and bulk modulus respectively. When both become negative simultaneously (where the material is said to be in its double negative (DNG) region), the refractive index has a negative sign so negative refraction occurs. In this region 
Snell's law still applies, however the path of the refracted wave lies to the opposite side of the incident normal than one would expect in a regular transmission medium, and the group and phase velocity vectors are anti-parallel. These novel properties mean that metamaterials are of particular interest and have been proposed as a potential solution to achieve acoustic cloaking [5] and subwavelength acoustic lenses [4]

This negative behaviour coincides with band gaps which appear in the dispersion characteristics of metamaterials where wave transmission is blocked. The locally resonant inclusions mean that these band gaps can be tuned to sub wavelength frequencies, meaning that they can be employed at frequencies much lower than traditional phononic crystals which rely on the Bragg scattering mechanism related to the periodic properties of the material. In traditional isolation scenarios, low frequencies are particularly troublesome, and therefore the ability to achieve high levels of wave attenuation at low frequencies across the material structure potentially makes metamaterials a powerful new tool in noise and vibration control [6]

Whilst the attenuation levels within the band gap are very high, the resonant nature of the band gap mechanism means that this attenuation occurs over a narrow bandwidth. Therefore, since noise issues are rarely narrowband and stationary in frequency, standard metamaterials are of limited use and it is necessary to change their properties to achieve broadband performance. A passive method for achieving a broader frequency band-gap is to stagger the natural frequencies of the resonant inclusions such that they each behave at independent, adjacent frequencies $[7,8]$. Whilst this does increase the bandwidth over which attenuation occurs it does so at the expense of the depth of the band-gap, i.e. the level of attenuation achieved, and can require a large number of resonators to cover a wide frequency band [7]. In addition, the frequency range at which passive solutions of this nature are useful remains fixed, and whilst adaptive solutions have been suggested $[9,10]$ the mechanical nature of these means their reaction speed is relatively slow, and their bandwidth remains limited by the number of resonators.

Recently research has been published incorporating the idea of active control within a metamaterial structure to enhance and control the resonant behaviour $[11,12,13,7,14]$ and whilst this work is in its infancy it is clear that this strategy has the potential to overcome the narrowband issues discussed above. So far much of this work has been focused on using local shunt circuits to tune active elements to achieve properties impractical using passive methods $[11,12,14]$. However recent work by Pope and Daley has investigated using multichannel fully active solutions that could increase metamaterial performance and be adapted online [13]. In this previous work there has been little experimental validation, and the active metamaterials that have been tested experimentally are far removed from practical exploitation. In this paper the feasibility of developing practically viable isolators using advanced manufacturing techniques is evaluated.

In the paper two materials are presented. First, a 1-dimensional acoustic metamaterial is developed consisting of split hollow spheres suspended in an acoustically transparent matrix. The split hollow sphere elements act as Helmholtz resonators 
and provide a region of wave attenuation and dispersive effective bulk modulus. The performance of the metamaterial is confirmed by experimental measurement within an impedance tube.

Then by using a lumped parameter mechanical analogy an elastodynamic equivalent is derived and developed. The inclusion of additional stiffness elements to couple the resonators to multiple transmission layers allows the material to achieve double negativity passively. The model is used as the basis of an experimental realisation of an active vibration isolator prototype. The passive performance is measured and the presence of resonant band gaps is confirmed. Active control is then employed in the material to broaden the region at which attenuation is produced using a Filtered-x Least Means Square (FxLMS) algorithm.

As metamaterials development continues towards a commercially viable solution it will be necessary to create a higher density of resonant elements and increase the degrees of freedom. As densities increase conventional manufacturing techniques are impractical on such scales, and additive layer manufacturing (3-dimensional printing) is likely to provide a solution. To evaluate the feasibility of using this as a manufacturing technique the materials presented here were designed for, and produced using, laser sintering methodologies.

\section{The acoustic metamaterial model}

The initial acoustic metamaterial considered is based on using an array of split hollow sphere (SHS) elements acting as Helmholtz resonators. Similar structures have been investigated by Ding et al [8]. SHS are analogous to the electromagnetic split ring resonators described by Pendry et al [15] and can be used to tune the effective bulk modulus of an acoustic material and create a band gap. This metamaterial provides a structure with which to validate the manufacturing technique and gain experimental results, as well as providing a basis for the design of the active vibration isolation prototype presented in Sections 5-8.

The resonant frequency of a Helmholtz resonator is $\omega_{0}=c_{0} \sqrt{\frac{S}{L^{\prime} V_{r}}}$ where $S$ and $L^{\prime}$ are respectively the cross sectional area and the effective length of the resonator neck, $c_{0}$ is the ambient sound speed and $V_{r}$ is the volume of air within the resonator cavity. When considering an array of SHS in an acoustic medium it is possible to derive an expression for the effective bulk modulus, $B_{e}$ of the material [6], where $M$ different types of resonators are combined within a metamaterial

$$
\frac{1}{B_{e}}=\frac{1}{B}\left(1-\sum_{m=1}^{M} \frac{F_{m} \omega_{0 m}^{2}}{\omega^{2}-\omega_{0 m}^{2}-j \Gamma \omega}\right)
$$

where $F_{m}$ is the volume ratio of the $m$ th SHS to the homogeneous medium, and $\Gamma$ is the intrinsic loss of the resonator elements. The wavenumber within the medium is defined as $k=\frac{\omega}{c}$ where $c$ is the frequency dependent sound speed, or phase velocity. The phase velocity of the effective medium is $c=\sqrt{B_{e} / \rho_{e}}$, where $\rho_{e}$ is the effective density. 


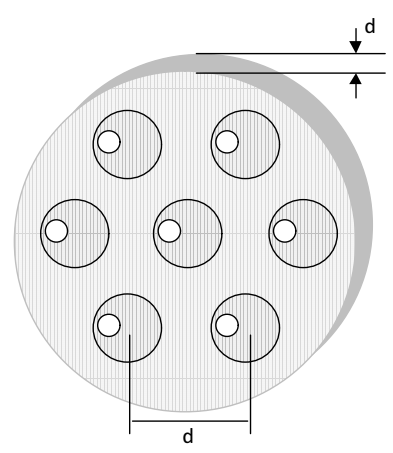

(a)

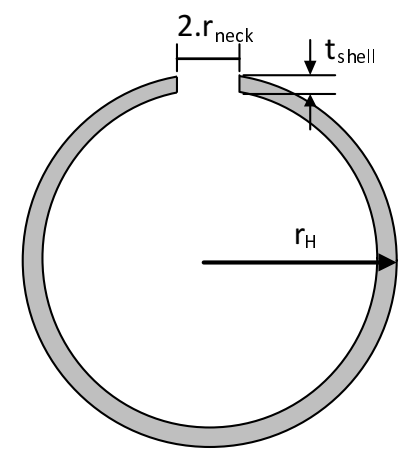

(b)

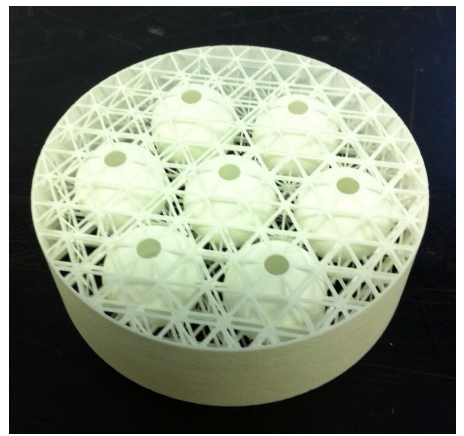

(c)

Figure 1. The schematic design of the metamaterial samples (a) the dimensions of the SHS resonators (b) and a photo of a produced metamaterial sample (c)

The metamaterial samples to be tested consist of varying numbers of independent layers, therefore a convenient method for modelling acoustic propagation through the material is the transfer matrix method. The relationship between the acoustic pressure, $P_{n}$ and particle velocity $U_{n}$ across a multiple layer material can be considered in terms of the transfer matrices, $\mathbf{T}_{n}$ such that

$$
\begin{aligned}
\left\{\begin{array}{l}
\mathbf{P}_{0} \\
\mathbf{U}_{0}
\end{array}\right\} & =\prod_{n=1}^{N} \mathbf{T}_{n} \cdot\left\{\begin{array}{l}
\mathbf{P}_{N+1} \\
\mathbf{U}_{N+1}
\end{array}\right\}=\boldsymbol{\tau}\left\{\begin{array}{l}
\mathbf{P}_{N+1} \\
\mathbf{U}_{N+1}
\end{array}\right\} \\
\mathbf{T}_{n} & =\left\{\begin{array}{ll}
\cos \left(k_{n} d_{n}\right) & i z_{n} \sin \left(k_{n} d_{n}\right) \\
\frac{i \sin \left(k_{n} d_{n}\right)}{z_{n}} & \cos \left(k_{n} d_{n}\right)
\end{array}\right\}
\end{aligned}
$$

where $T_{n}$ is the transfer matrix of a single homogeneous layer, and $k_{n}$ is dependent on the phase velocity, $c_{n}$, of the $n$th layer. Note that if the multiple layers are identical, as is the case with a periodic metamaterial, the product of transfer matrices, $\boldsymbol{\tau}$, reduces to $\mathbf{T}^{N}$. This transfer matrix principle is used to model the dynamic response of the acoustic metamaterial and compare it to measurements, and is also adapted and applied to the elastodynamic metamaterial presented in Section 5

\section{Acoustic Metamaterial Experimental Sample Design}

The form of the acoustic metamateiral has been chosen so their performance can be readily tested within an impedance tube and the transmission loss and effective bulk modulus measured [16]. The designs consist of individual circular samples of a triangular porous mesh containing a hexagonal array of 7 SHS hollow spheres, a schematic of the design and a photograph of a produced sample are shown in Figure 1. The mesh provides structural integrity only, and is designed to be acoustically transparent. The samples have a thickness, $T$, and lattice constant, $A$, of $30 \mathrm{~mm}$. One of the future 


\begin{tabular}{|l|l|l|l|l|}
\hline Type & $2 r_{\text {neck }}, \mathrm{m}$ & $r_{H}, \mathrm{~m}$ & $t_{\text {shell }}, \mathrm{m}$ & $\omega_{0} /(2 \pi), \mathrm{Hz}$ \\
\hline $\mathrm{A}$ & 0.0030 & 0.0125 & 0.0007 & 960 \\
\hline $\mathrm{B}$ & 0.0040 & 0.0125 & 0.0007 & 1145 \\
\hline $\mathrm{C}$ & 0.0060 & 0.0125 & 0.0007 & 1451 \\
\hline
\end{tabular}

Table 1. Dimensions of the metamaterial samples

aims of this research is to produce metamaterials that have lattice constants on a micro scale where one of the only viable production techniques is likely to be currently emerging 3-dimensional printing technologies. Therefore the samples have designed to be produced out of Polyamide 6, using existing 3D printing technology. In all four types of metamaterial sample were specified; three structures with arrays of SHS resonators of differing neck diameter, $d$, (resonator types A-C, with resonant frequencies of $960 \mathrm{~Hz}, 1145 \mathrm{~Hz}$ and $1451 \mathrm{~Hz}$ respectively) and one structure with mixed resonators (two resonators of type $\mathrm{A}$, three of type $\mathrm{B}$, two of type $\mathrm{C}$ ). The resonator dimensions are described in Table 1. In addition two control structures were specified; one a plain mesh with no SHS elements, and one with an array of solid (non resonant) spheres.

\section{Acoustic Metamaterial Experimental Results}

\subsection{Transmission}

Using the transfer matrix approach it is clear that as more layers of metamaterial are added the transfer matrix, $\boldsymbol{\tau}$, is raised to a higher power such that $\boldsymbol{\tau}=\boldsymbol{T}_{n}^{N}$. Therefore, as more layers are added it would be expected that the depth of the band gap increases but the edge frequencies to remain constant. Figure 2(a) confirms that this behaviour occurs in both simulations and measured results. For the theoretical results presented, the intrinsic losses of the resonator elements, $\Gamma$, was chosen to best fit the measured results. Conversely, changing the natural frequency of the resonator elements leads to a change in the location of the resulting band gap. Figure 2(b) shows how the band gap changes location as the resonant frequency of the resonators is changed for both simulated and measured results. As a datum, mesh structures containing no resonator elements were also measured, as well as mesh structures containing solid spheres. Here the lack of resonant behaviour means that the transmission coefficient is almost unity, proving the effects seen are not due to scattering of the mesh or the spheres.

\subsection{Wave number and bulk modulus}

Figures 2(c) and 2(d) show the simulated and measured bulk modulus and wave number of 3 layers of a metamaterial with $4 \mathrm{~mm}$ type resonators. The gradient of the real part of the wave number (i.e. the group velocity) becomes negative at the resonant frequency of the SHS elements, a result of the dispersive bulk modulus shown. An increase in the magnitude of the imaginary part of the wave number results in attenuation 

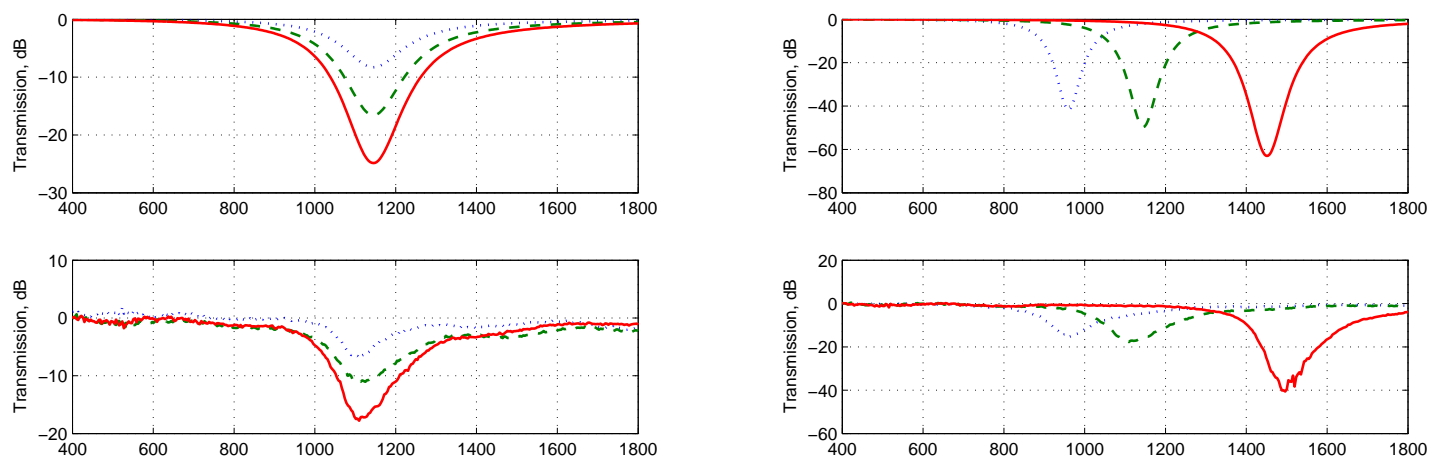

(a)

(b)
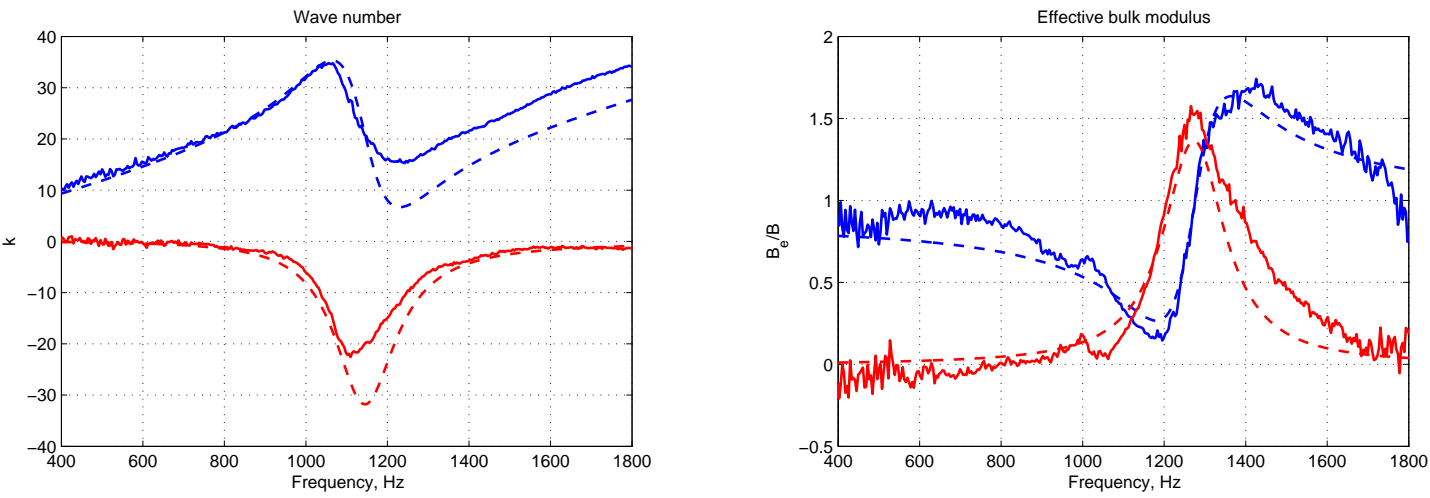

(c)

(d)

Figure 2. (a) The transmission behaviour of 1 (dotted), 2 (dashed) and 3 (solid) layers of the $4 \mathrm{~mm}$ resonator metamaterial, simulated (top) and measured (bottom). (b) The transmission behaviour of 2 layers of $3 \mathrm{~mm}$ (dotted), $4 \mathrm{~mm}$ (dashed) and $6 \mathrm{~mm}$ (solid) resonator metamaterial, simulated (top) and measured (bottom). The wave number (c) and bulk modulus (d) of a 3 layer metamaterial with $4 \mathrm{~mm}$ type resonators, real (blue) and imaginary (red). The simulated (dotted) and measured (solid) results are presented

which creates the band gap. The bulk modulus for the control samples is shown to be approximately the ambient value for air $\left(B_{0}\right)$ and the wave number to increase linearly with frequency, as expected. The bulk modulus is shown to have a dip around resonance, but in most cases the measured dip is not enough to overcome the static bulk modulus of the transmission medium and achieve negativity. The effective bulk modulus and wave number remains constant with the addition of extra layers of metamaterial as it is function of the ratio of the resonator volumes to the overall volume of the metamaterial, which remains constant. Therefore negativity could theoretically be achieved by changing the dimension/number of resonators to increase the volume ratio, $F$, and allow the dispersive bulk modulus component to become larger and overcome the static component. 


\subsection{Mixed Resonator Samples}

Although not presented in the results here, when the mixed resonator samples are tested three separate band gaps appear at the three resonator frequencies. The measurements also show multiple dips in the effective bulk modulus and the wave number of the material at the separate resonant frequencies. These results mirror those produced by Ding et al [8], and with more resonators with frequencies spaced close together it would be possible to achieve a broader static band gap. The results here show that such a design could be realised practically through the deployment of additive layer manufacturing.

\section{Elastodynamic Metamaterial Design}

Within the long wavelength limit it is possible to apply a mechanical analogy to the acoustic elements described above and build a lumped parameter mass-spring-damper model of the system. A Helmholtz resonator can be modelled by as a simple mass element attached to a spring-damper representing the mass of the air within the resonator neck and the viscoelastic properties of the air within the cavity respectively. An acoustic duct can then be modelled as a simple chain of masses and spring-dampers. Therefore, considering a metamaterial such as the SHS examples above and taking the simple case of a single resonator per layer, the multi-layer material can be modelled as the lumped parameter example given by Figure 3(a), where $m_{t}, k_{t}$ and $c_{t}$ represent the mass, stiffness and damping of the air within the duct, referred to as the transmission medium, and $m_{r}, k_{r}$ and $c_{r}$ represent the mechanical properties of the resonators. By adding active point forces to the resonator masses, $f_{n}$, the material mirrors that first

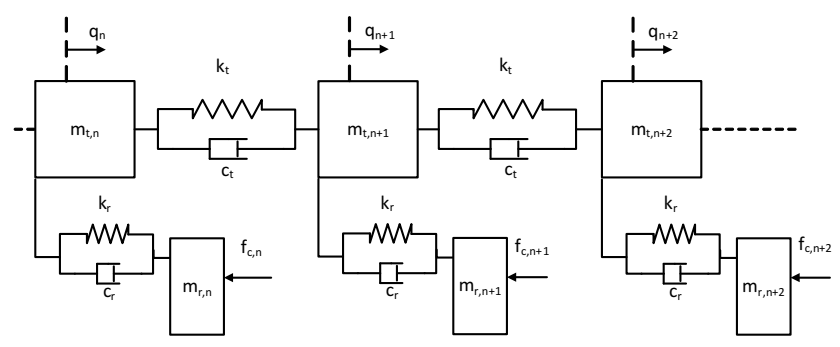

(a)

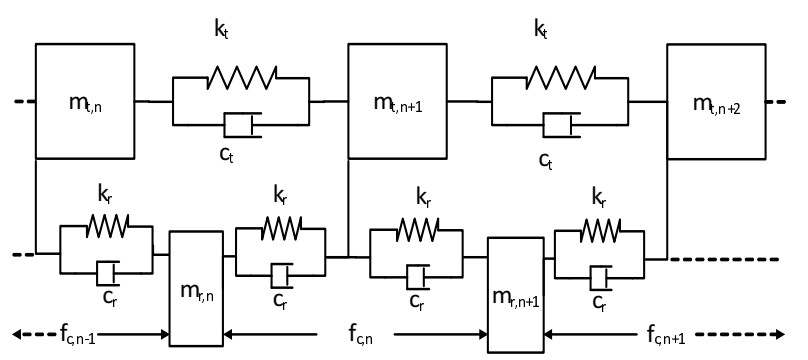

(b)

Figure 3. (a) A mechanical analogue of the duct-resonator material with active forces applied (c) The passively coupled metamaterial model 
presented by Pope and Daley [13] where it was shown that such a material would be single negative, with negative effective mass. Pope and Daley showed that to achieve double negativity within the material the active force must be used to couple the motion of the resonators with adjacent layers.

However producing and applying such a material leads to some practical problems, and therefore the material presented here the active metamaterial model has been developed further. Using inertial actuators to deliver point forces, as required by the original design, can lead to complications due to the nature of the actuator dynamics. Therefore the active architecture has been changed to incorporate reactive forces rather than point forces, thus simplifying the control design process at the expense of restricting the nature of forces that can be applied.Also, additional passive viscoelastic connections have been added coupling each resonator mass to two transmission masses, which enables the material to become double negative passively. The resulting material is shown in Figure 3(b).

Such a material can be modelled as a simple chain of equivalent masses, $M_{e}$, connected by equivalent dispersive viscoelastic connections $K_{e}+j \omega C_{e}$ [13] such that the equations of motion reduce to

$$
-\omega^{2} M_{e} x_{n}+\left(i \omega C_{e}+K_{e}\right)\left(2 x_{n}-x_{n+1}-x_{n-1}\right)=f_{n}
$$

Formulating and rearranging the equations of motion for the explicit material model in Figure 3(b), gives

$$
\begin{aligned}
& -\omega^{2}\left(m+\frac{2 m_{r}\left(k_{r}+j \omega c_{r}\right)}{-\omega^{2} m_{r}+2\left(k_{r}+j \omega c_{r}\right)}\right) x_{n} \ldots \\
& \quad+\left(k+j \omega c+\frac{\left(k_{r}+j \omega c_{r}\right)^{2}}{-\omega^{2} m_{r}+2\left(k_{r}+j \omega c_{r}\right)}\right)\left(2 x_{n}-x_{n+1}-x_{n-1}\right)=f_{n}
\end{aligned}
$$

From this it is evident that the effective mass, $M_{e}$ and effective stiffness and damping $K_{e}+j \omega C_{e}$ are given by

$$
\begin{gathered}
M_{e}=m+\frac{2 m_{r}\left(k_{r}+j \omega c_{r}\right)}{-\omega^{2} m_{r}+2\left(k_{r}+j \omega c_{r}\right)} \\
K_{e}+j \omega C_{e}=k+j \omega c+\frac{\left(k_{r}+j \omega c_{r}\right)^{2}}{-\omega^{2} m_{r}+2\left(k_{r}+j \omega c_{r}\right)}
\end{gathered}
$$

These expressions are complex, where $\operatorname{Im}\left(M_{e}\right)$ is an additional dissipative term introduced by the presence of damping within the resonator elements. $\operatorname{Re}\left(M_{e}\right)$ is the true effective inertial mass of the system and the quantity of interest. Likewise the real part of $K_{e}+j \omega C_{e}$ is the effective stiffness of the system, with the imaginary part being the dissipative effective damping. The expressions for effective stiffness and mass have a resonance at the same frequency, and hence become negative at the same time making the material double negative. 
As for the acoustic periodic metamaterial from Section 2, a transfer matrix approach is useful for modelling the dynamics of such a material and a typical passive material transmission response (the motion of the last transmission mass related to the first), including the sign of each effective material parameter is shown in Figure 4(a). The transfer matrix for the elastodynamic material is given by

$$
\boldsymbol{T}_{n}=\left[\begin{array}{cc}
1-\frac{\omega^{2} M_{e}}{K_{e}+j \omega C_{e}} & -\frac{1}{K_{e}+j \omega C_{e}} \\
\omega^{2} M_{e} & 1
\end{array}\right]
$$

\section{Dispersion Relationship}

As Figure 4(a) shows, the double negative region also coincides with the region of attenuation commonly referred to as the band gap. Previous studies have suggested that the resonant band gap occurs in a single negative region where the wave vector, $k$, becomes imaginary, since this leads to an evanescent rather than travelling wave solution to the wave equation. When the material is made to be double negative, the solution becomes negative real and a passband occurs in what is now a 'left-handed' material [17]. However, this explanation neglects dissipative losses within the material. Equation 9 describes the displacement of the $n$th element of the periodic material in relation to its preceding cell

$$
x_{n}=e^{j k d} x_{n-1}
$$

Where $k$ is the wave vector and $d$ is the lattice constant of the material. Substituting this expression into Equation 4 yields the Bloch dispersion relationship of a the periodic

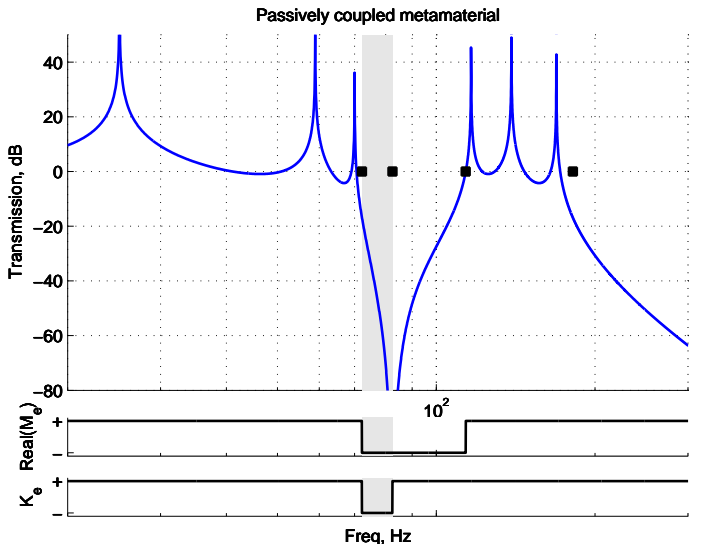

(a)

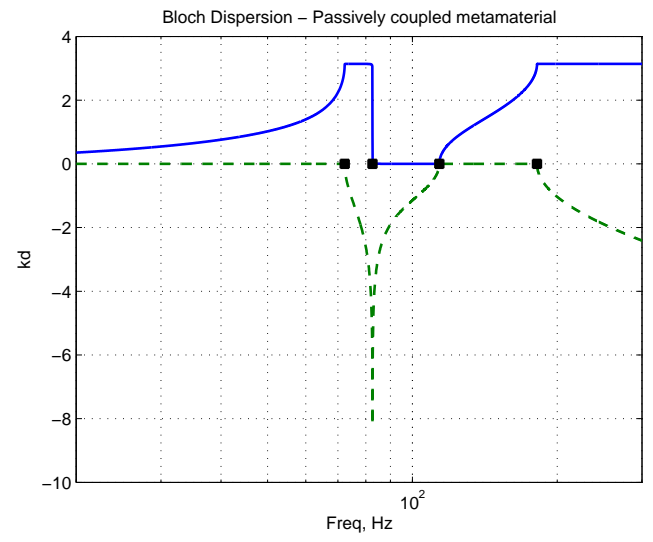

(b)

Figure 4. (a) The passive transmission response of a 4 layer coupled metamaterial, the double negative region (shaded), and 4 break frequencies of the dispersion relationship (black dots). (b) The dispersion relationship of the material, real (solid) and imaginary (dashed) 
metamaterial of infinite length

$$
\cos k d=1-\frac{\omega^{2} M_{e}}{2\left(K_{e}+j \omega C_{e}\right)}
$$

Here even for a DNG material, if the effective material parameters are complex themselves, the result will be complex leading to a travelling (real) wave with an attenuation (imaginary) envelope. When the relationships governing the effective material parameters resonate, the magnitude of imaginary terms become very large, leading to high levels of attenuation.

In fact, the wave number will become complex even in the absence of dissipative elements when $|\cos k d|>1$. This occurs when $\alpha>2$ or $\alpha<0$, where $\alpha=\omega^{2} M_{e} /\left(2 K_{e}\right)$. Considering these conditions, combining Equations 10, 6 and 7 leads to 3 inequalities that define the band gap. $\alpha$ will be positive only if both the numerator and denominator are of the same sign, i.e. if both of the following inequalities are simultaneously true or false

$$
\begin{aligned}
\omega^{2}<\frac{\left(m_{t}+m_{r}\right) k_{r}}{m_{t} m_{r}} \\
\omega^{2}<\frac{\left(2 k_{t}+k_{r}\right) k_{r}}{k_{t} m_{r}}
\end{aligned}
$$

These expressions represent the numerator and denominator respectively. The third inequality defines where $\alpha>2$ and can be written as a quadratic expression in $\omega^{2}$

$$
-\omega^{4} m_{t} m_{r}+\omega^{2}\left(2\left(m_{t}+m_{r}\right) k_{r}+4 m_{r} k_{t}\right)-4\left(k_{r}\left(2 k_{t}+k_{r}\right)\right)>0
$$

which has two positive solutions

$$
\begin{gathered}
\omega_{0, r}^{2}=\frac{2 k_{r}}{m_{r}} \\
\omega_{U L}^{2}=\frac{2 k_{r}+4 k_{t}}{m_{t}}
\end{gathered}
$$

Combining 14 with the inequalities 11 and 12 yields two more break frequencies

$$
\begin{aligned}
& \omega_{\text {mid }}^{2}=\omega_{0, r}^{2}\left(1+\frac{k_{r}}{2 k_{t}}\right) \\
& \omega_{\text {end }}^{2}=\omega_{0, r}^{2}\left(1+\frac{m_{r}}{m_{t}}\right)
\end{aligned}
$$

Both the numerator and denominator are positive as $\omega \rightarrow 0$, which allows the use of these four frequencies to describe the dynamics of the material and, crucially, determine the position and width of the band gap. $\omega_{0, r}$ would be the natural frequency of the resonator elements if they were traditional vibration absorbers, and is the point at which (13) becomes true, denoting the lower edge of the band gap. As $\omega$ increases the left hand side of (13) becomes larger as the denominator governed by (12) becomes 
smaller and attenuation increases. At $\omega_{\text {mid }}$ the denominator becomes zero, $\omega^{2} M_{e} /\left(2 K_{e}\right)$ resonates and the band gap attenuation peaks. As $\omega$ increases further the denominator (12) becomes negative, $\alpha$ becomes negative and $k d$ remains complex. Now the magnitude of the denominator $2 K_{e}$ gets larger, reducing the magnitude of the $\alpha$ and limiting band gap attenuation. Finally, at $\omega_{\text {end }}$, the numerator inequality (11) becomes false and the wave number becomes pure real, denoting the upper band gap edge. The fourth break frequency, $\omega_{U L}$, defines the dynamic limit of the lumped parameter system, above which response rolls off as a low pass mechanical filter, and is independent of the resonator mass. From the above expressions it is clear that it is possible to adjust the total width of the band gap by adjusting the ratio of the resonator and transmission masses.

Note, that in Figure 4(a) there is a small discrepancy between $\omega_{0, r}$ and the onset of the band gap and $\omega_{U L}$ and the onset high frequency roll off. This is due to the finite nature of the 4 layer model plotted. Bloch wave number analysis presumes an infinite number of material layers. In addition, due to the cross coupling of the material presented here, the effective material parameters of the first and last layers differ slightly from Equations 6 and 7 and effect the transmission performance. As the number of layers, $N$, increases these onset frequencies tend towards the calculated values given by Equations 14 and 15.

Plotting $k d$ reveals the dispersion characteristics of the material, Figure 4(b), and it is clear that the region where the magnitude of the imaginary part of the wave number becomes large coincides with the band gap of the material response. Note that due to the restricted domain of the $\cos ^{-1}$ function, the calculated real part of the dispersion characteristic is restricted to $0 \leq k d \leq \pi$; the physical wave number is not bound in this way. The black dots on Figure 4(a) and 4(b) represent the 4 break frequencies described above. Note that the first two break frequencies coincide with the edges of the double negative region.

\section{Elastodynamic Metamaterial Experimental Design}

To investigate the practical viability of the elastodynamic metamaterial design, a 4-layer experimental realisation of the metamaterial was designed $\ddagger$, consisting of titanium discs separated by coil springs acting as the transmission medium. Between each transmission layer is a resonator mass, connected to each transmission layer using leaf springs. The

material was produced as 3 cells using an additive layer, selective laser melting (SLM) technique, so each cell is a discrete titanium structure. The cells then bolt together to produce a continuous, periodic material. The resonator masses contain hollows to accommodate voice coil type reactive actuators, such that control forces can be applied as per Figure 3(b). A CAD representation of the cell design is shown in Figure 5(a), alongside a photo of the assembled material in Figure 5(b).

To measure the response of the metamaterial the structure is bolted to a steel plate which is then excited using an inertial actuator. The motion of the top and bottom

$\ddagger$ Note: The active metamaterial presented here is subject to a patent (pending) number GB1304500.0 


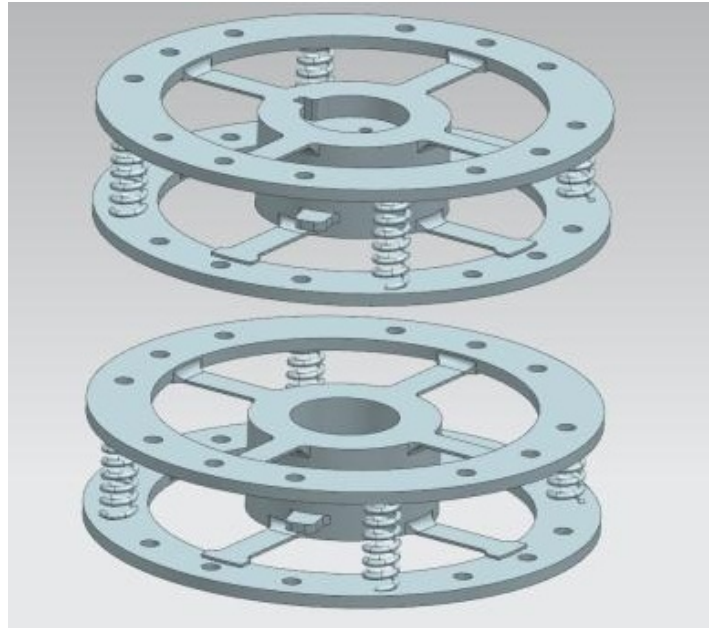

(a)

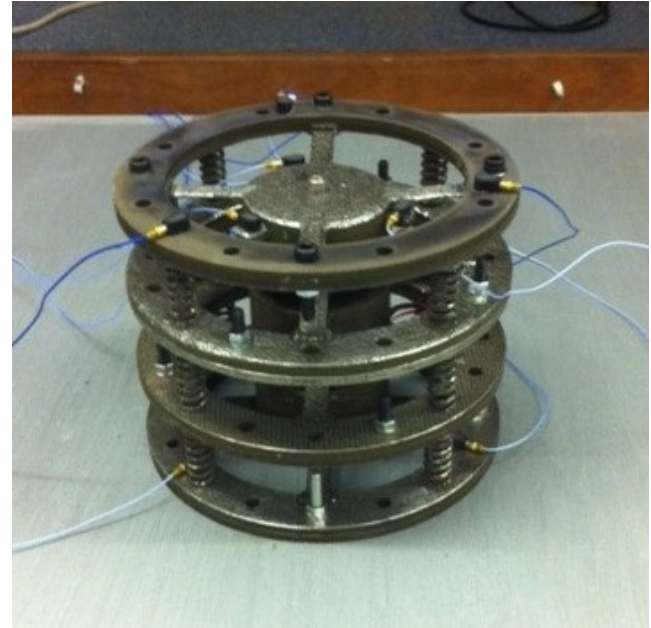

(b)

Figure 5. (a) A 3D CAD representation of the metamaterial construction. (b) A photo of the experimental metamaterial

transmission discs is then measured using accelerometers and the transfer function calculated. A dSpace rapid prototyping system is used to provide the excitation signal as well as record the accelerometer signals.

\section{Elastodynamic Metamaterial Experimental Results}

\subsection{Passive Metamaterial Response}

Figure 6(a) presents the passive response of the material for different excitation levels, as well as the coherence between the signals measured at the bottom and top plate. The material was designed to have a passive band gap at $87 \mathrm{~Hz}$, and at lower excitation levels there does appear to be attenuation in this region, however this attenuation decreases as the excitation level increases. Therefore this behaviour is the consequence of a nonlinearity, thought to be due to the presence of stiction within the actuator assemblies due to misalignment as well as a significant air-spring effect. However, it can be seen from the response that there are stable band gaps at higher frequencies - A deep, narrow gap at $207 \mathrm{~Hz}$, and a wider but shallow gap around $250 \mathrm{~Hz}$. During the manufacturing process it became clear that the additive layer manufacturing technique employed was being stretched to its limitations, and several problems had to be overcome. In light of this the discrepancy in the specified and resulting response is perhaps not surprising. Of particular consequence is the specification of the leaf springs, the stiffness of which is very sensitive to the thickness, $t_{b}$ of the springs $\left(k_{r} \propto t_{b}^{3}\right)$; manufacturing difficulties meant that the leaf springs produced were of varying thickness, and some springs were warped and do not sit parallel with the transmission plate. This is likely to have had a large effect on the stiffness of the elements and means that the 2 resonator elements are not resonating at the designed frequency. However, the presence of a resonant band 

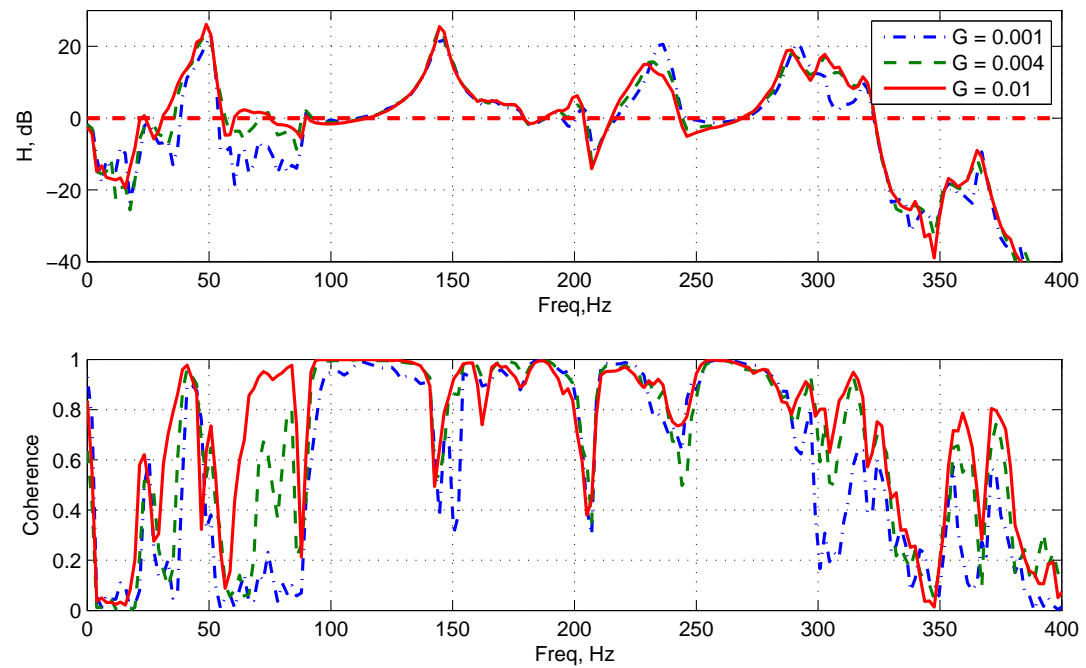

(a)
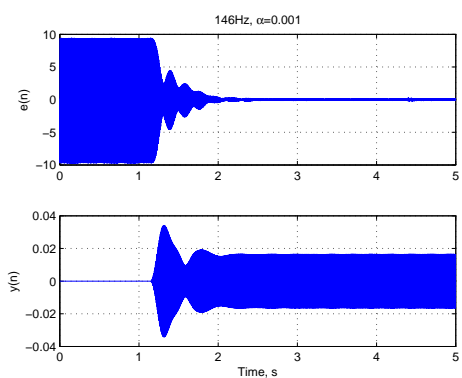

(b)

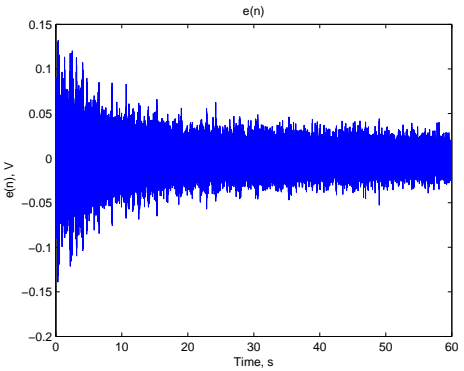

(c)

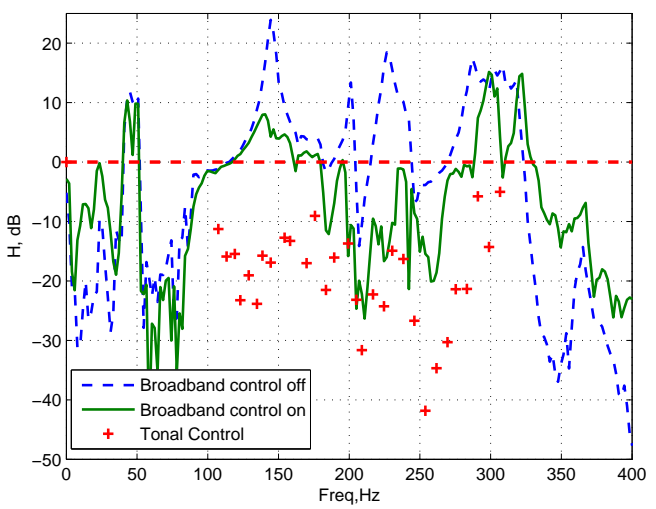

(d)

Figure 6. (a) Transmission spectrum of the metamaterial at varying levels of input disturbance (top) and related coherence (bottom) for increasing input gain. (b-c) Time history of the active metamaterial when control is applied after 1 second, where (b) shows the top transmission plate acceleration (top) and control signal (bottom) for a $146 \mathrm{~Hz}$ tonal disturbance and (c) the acceleration of the top transmission plate for a band limited broadband disturbance. (d) The passive band limited broadband transmission performance (dashed), closed loop response (solid) with the equivalent maximum reduction achieved to a tonal input at each frequency $(+)$ 
gap at the higher frequencies means that the material does validate the potential of the approach and provide a useful tool to investigate the application of active control to enhance the band gap profile.

\subsection{Applying active control to the metamaterial}

The dSpace system can also apply active control, processing the accelerometer signals in real time and applying control forces to the reactive actuators. For these initial investigations a single channel Filtered-x Least Mean Squared (FxLMS) algorithm was used as this is a well established, robust algorithm [18] that could be simply implemented to demonstrate the efficacy of the active approach. The algorithm was employed to minimise the acceleration of the top transmission plate using the acceleration of the bottom plate as a reference signal. Although the active material contains two actuators, in this instance only one is employed.

Several measurements were carried out to apply the algorithm to control tonal disturbances as well as a white noise disturbance that was band limited from $100 \mathrm{~Hz}$ to $300 \mathrm{~Hz}$. Figures 6(b) and 6(c) show time histories for the top transmission plate after control has been applied for both tonal and broadband disturbances respectively. The controller is able to achieve a considerable reduction in the transmission relative to the passive performance. The tonal control is achieved using two filters of 1 coefficient, controlling the amplitude of an in-phase and a quadrature version of the reference signal to cancel out the disturbance. To control a broadband disturbance, a single control filter of 2048 coefficients is shaped by the FxLMS algorithm.

Figure $6(\mathrm{~d})$ shows the transmission spectrum of the passive material alongside the performance achieved by the active material when subject to the band limited broadband disturbance. Also plotted on the graph are the equivalent reductions achieved by the tonal controller at each distinct frequency. The performance of the broadband controller is limited by the length of the control filter, the maximum force output of the actuator, and a causal filter constraint that will apply if the speed of the control system is slower than wave propagation across the material. The tonal results can be considered a guide to the maximum performance that could be achieved using this set up, either using a longer filter or using more refined broadband control algorithm. The broadband results demonstrate that over the frequency span where excitation is present there is a significant improvement over the passive isolation performance. The naturally occurring band gap has been significantly broadened, and peak attenuation levels have been enhanced significantly. Detrimental out of band resonances have also been suppressed, notably at $146 \mathrm{~Hz}$.

The tonal results show that significant isolation performance can be achieved over a broad frequency range, in some cases up to 40dB. Of significance is the fact that the greatest levels of attenuation are achieved where the natural band gaps occur. This supports the argument for using active periodic materials in vibration isolation applications over more traditional active isolation mounts. A unified design process that 
matches the best features of both active and passive functionality can provide impressive levels of attenuation over wide band widths. Despite the fact that the experimental metamaterial did not match the original design specification, the results demonstrate a proof of concept that active architecture can be used to enhance the passive performance of metamaterials, and in particular the narrowband behaviour inherent in periodic, locally resonant designs.

\section{Conclusions}

A common metamaterial structure is a periodic array of Helmholtz resonators within an acoustic duct. Such a metamaterial was designed by suspending split hollow spheres within an acoustically transparent matrix. Using additive layer manufacturing to produce these materials, attenuation and the presence of dispersive effective bulk modulus within the band gap region was demonstrated. By applying an acoustic/mechanical analogy this type of metamaterial was used as a basis to design an elastodynamic metamaterial and by applying additional viscoelastic elements to the model this material was shown to exhibit double negative behaviour passively. Active control architecture was incorporated into the model and this used to design a prototype active isolator produced using additive layer manufacturing. The elastodynamic material was shown to exhibit band gap behaviour and furthermore is was demonstrated that this band gap could be significantly enhanced using active control, and that the combination of a passive band gap with active enhancement leads to high levels of isolation performance. This work demonstrates how the combination of metamaterial design, active functionality and modern 3-dimensional printing manufacturing could lead to high performance compact devices for isolation applications.

\section{Acknowledgements}

The metamaterials were produced by the EPSRC National Centre for Innovative Manufacturing in Additive Manufacturing, Nottingham University. The assistance of Dr Chris Tuck and Prof. Richard Hague is gratefully acknowledged. The authors would also like to acknowledge the helpful collaboration with Dr Simon Pope of the University of Sheffield. This work was funded by the Engineering and Physical Science Research Council (EPSRC) and BAE Systems under a Collaborative Award in Science and Engineering (CASE) award, with additional funding from the Defence Science and Technology Laboratory (DSTL) MAST programme 23804

\section{Bibliography}

[1] D. R. Smith and N. Kroll. Negative refractive index in left-handed materials. Physical Review Letters, 85(14):2933-2936, 2000.

[2] V. Veselago. The electrodynamics of substances with simultaneously negative values of permittivity and permeability. Soviet Physics Uspekhi-Ussr, 10(4):509-514, 1968. 
[3] N. Fang, D. J. Xi, J. Y. Xu, M. Ambati, W. Srituravanich, C. Sun, and X. Zhang. Ultrasonic metamaterials with negative modulus. Nature Materials, 5(6):452-456, 2006.

[4] Z. Y. Liu, X. X. Zhang, Y. W. Mao, Y. Y. Zhu, Z. Y. Yang, C. T. Chan, and P. Sheng. Locally resonant sonic materials. Science, 289(5485):1734-1736, 2000.

[5] H. Y. Chen and C. T. Chan. Acoustic cloaking and transformation acoustics. Journal of Physics D-Applied Physics, 43(11), 2010.

[6] Y. Cheng, J. Y. Xu, and X. J. Liu. Broad forbidden bands in parallel-coupled locally resonant ultrasonic metamaterials. Applied Physics Letters, 92(5), 2008.

[7] M. Reynolds, S. Daley, Y. Gao, V. Humphrey, and S. A. Pope. Controller architectures for optimum performance in practical active acoustic metamaterials. Proceedings of Acoustics 2012: 11th Congres Francais d'Acoustique and 2012 IOA annual meeting. Nantes, France, 2012.

[8] C. L. Ding and X. P. Zhao. Multi-band and broadband acoustic metamaterial with resonant structures. Journal of Physics D-Applied Physics, 44(21), 2011.

[9] K. J. B. Lee, M. K. Jung, and S. H. Lee. Highly tunable acoustic metamaterials based on a resonant tubular array. Physical Review B, 86(18), 2012.

[10] L. M. Hao, C. L. Ding, and X. P. Zhao. Tunable acoustic metamaterial with negative modulus. Applied Physics a-Materials Science $\mathcal{G}$ Processing, 106(4):807-811, 2012.

[11] A. M. Baz. An active acoustic metamaterial with tunable effective density. Journal of Vibration and Acoustics-Transactions of the Asme, 132(4), 2010.

[12] W. Akl and A. Baz. Configurations of active acoustic metamaterial with programmable bulk modulus. Active and Passive Smart Structures and Integrated Systems 2010, Pts 1 and 2, 7643, 2010.

[13] S. A. Pope and S. Daley. Viscoelastic locally resonant double negative metamaterials with controllable effective density and elasticity. Physics Letters A, 374(41):4250-4255, 2010.

[14] L. Airoldi and M. Ruzzene. Design of tunable acoustic metamaterials through periodic arrays of resonant shunted piezos. New Journal of Physics, 13, 2011.

[15] J. B. Pendry, A. J. Holden, D. J. Robbins, and W. J. Stewart. Magnetism from conductors and enhanced nonlinear phenomena. Ieee Transactions on Microwave Theory and Techniques, 47(11):2075-2084, 1999.

[16] B.H. Song and J.S. Bolton. A transfer-matrix approach for estimating the characteristic impedance and wave numbers of limp and rigid porous materials. The Journal of the Acoustical Society of America, 107:1131, 2000.

[17] S. H. Lee, C. M. Park, Y. M. Seo, Z. G. Wang, and C. K. Kim. Composite acoustic medium with simultaneously negative density and modulus. Physical Review Letters, 104(5), 2010.

[18] S. Elliott. Signal Processing for Active Control. Elsevier Science, 2000. 